\title{
EQUIVALENCE OF NORMS FOR COEFFICIENTS OF UNITARY GROUP REPRESENTATIONS ${ }^{1}$
}

\author{
ALBERTO ALESINA ${ }^{2}$
}

\begin{abstract}
We study the equivalence between the $B(G)$ norm and the supremum norm in the Banach space $A_{\pi}$, the closed subspace of $B(G)$ which is generated by the coefficients of a continuous, unitary, irreducible, infinite dimensional representation $\pi$ of a locally compact, noncompact group $G$.
\end{abstract}

1. Introduction. Let $B(G)$ be the Fourier-Stieltjes algebra of a locally compact group $G$ with the usual norm:

$$
\|u\|_{B}=\sup \left|\int_{G} f(x) u(x) d x\right|: f \in L^{1}(G), \quad\|f\|_{C^{*}(G)} \leqslant 1,
$$

$C^{*}(G)$ being the $C^{*}$ algebra of the group $G$; let $A_{\pi}$ be the closed subspace of $B(G)$ generated by the coefficients $\left\{(\pi(x) w, z): w, z \in H_{\pi}\right\}$ of a unitary representation $\pi$ of $G$ on a Hilbert space $H_{\pi}$ with inner product $(\cdot, \cdot)$ [1], [2], [3].

We are interested in comparing the $B$ norm and the supremum norm on this subspace. If the representation $\pi$ is contained in the left regular representation of $G$ and $G$ is abelian and compact, then the equivalence of the two norms on $A_{\pi}$ is equivalent to the support of $\pi$ being a Sidon set in the dual group of $G$ [4, Theorem 5.7.3], [2, (2.13)]. Sidon sets have also been studied in the context of nonabelian compact groups.

In the noncompact, nonabelian case, even the problem whether for a single infinite dimensional irreducible representation the two norms are equivalent or not on $A_{\pi}$ seems to be a nontrivial one. Although we are not able to give any necessary and sufficient condition, we prove that for a fairly large class of such representations the norms are inequivalent.

On the other hand we give a sufficient condition for the equivalence, and we provide an example in which this phenomenon occurs. Our results are contained in the following theorems:

Received by the editors March 21, 1978.

AMS (MOS) subject classifications (1970). Primary 22D10; Secondary 22D30.

'Written while the author was visiting the University of California, Berkeley.

${ }^{2}$ Supported in part by the National Research Council of Italy. 
THEOREM 1. If the subgroup $\pi(G)$ of the unitary group $\mathcal{U}$ on $H_{\pi}$ satisfies the condition:

"there exists a $\delta>0$ such that for every two finite orthonormal systems $\left\{w_{i}\right\},\left\{z_{i}\right\},(i=1, \ldots, k)$ in $H_{\pi}$ and for every unitary operator $U \in \mathcal{Q}$, there exists an operator $U^{\prime} \in \pi(G)$ such that:

$$
\left|\left(\left(U-U^{\prime}\right) w_{i}, z_{i}\right)\right|<(1-\delta) \quad(i=1, \ldots, k) "
$$

then the $B$ norm and the supremum norm are equivalent on $A_{\pi}$.

THEOREM 2. If all the coefficients of the representation $\pi$ vanish at infinity (possibly modulo the kernel of the representation), then the norms are inequivalent on $A_{\pi}$.

THEOREM 3. Let $\pi$ be a representation of $G$ which is induced by a representation $\rho$ of a closed subgroup $N$ such that the homogeneous space $G / N$ is not discrete. Assume that:

either $G / N$ has a $G$-invariant measure,

or $N$ is compact and $G / N$ has a relatively invariant measure;

then the two norms are inequivalent in $A_{\pi}$.

We recall that the representation $\pi$ is always assumed irreducible. For theorems and terminology about induced representations we will refer to [5].

We denote by $\mathcal{L}(H)$ the algebra of all the bounded linear operators on a Hilbert space $H$, and by $\mathcal{T}(H)$ the ideal of the trace class operators with norm: $\|T\|_{1}=\operatorname{tr}(|T|)$, where $|T|$ is the absolute value of $T$ and $\operatorname{tr}$ is the canonical, faithful, normal semifinite trace on $\mathcal{L}(H)^{+}$. We recall that $\mathcal{L}(H)$ can be identified with the dual of $\mathcal{T}(H)$ via the pairing

$$
\langle L, T\rangle=\operatorname{tr}(L T), \quad L \in \mathcal{L}(H), T \in \mathcal{T}(H)[6] .
$$

We will make use of the following characterization of $A_{\pi}$, for $\pi$ irreducible, which can be easily deduced from [7, Theorem 3].

Proposition 1. $A_{\pi}$ is isometrically isomorphic to $\mathcal{T}\left(H_{\pi}\right)$ via the so-called "inverse Fourier transform localized at $\pi$ ":

$$
t(x)=\operatorname{tr}(\pi(x) T), \quad x \in G, T \in \mathcal{T}\left(H_{\pi}\right) ;
$$

i.e. every function in $A_{\pi}$ is of the form $t$ for some $T \in \mathcal{T}\left(H_{\pi}\right)$, the correspondence is one-to-one and $\|t\|_{B(G)}=\|T\|_{1}$.

We thank Professor A. Figà-Talamanca for suggesting this problem to us and Professor L. DeMichele for his useful advice.

2. Proof of Theorem 1. We prove a more general result for any subgroup $\mathfrak{V}$ of the unitary group $\mathscr{Q}$ of the Hilbert space $H_{\pi}$ satisfying the hypothesis of the theorem. Namely, if for such subgroup we define

$$
n(T)=\sup |\operatorname{tr}(U T)|: U \in \mathcal{T},
$$


then

$$
\delta\|T\|_{1} \leqslant n(T) \leqslant\|T\|_{1}, \quad \text { for every } T \in \mathcal{T}\left(H_{\pi}\right) .
$$

The theorem will follow by Proposition 1 , choosing $\mathscr{V}=\pi(G)$ so that $n(T)=\|t\|_{\infty}$.

It is enough to prove the left-hand side inequality for all the finite rank operators, which are dense in $\mathcal{T}\left(H_{\pi}\right)$ with respect to the trace norm. The right-hand side inequality is trivial. Without loss of generality let the operator $T$ be defined by:

$T w=\sum_{i=1}^{k} \lambda_{i}\left(w, z_{i}\right) w_{i}, w \in H_{\pi}, \lambda_{i}$ complex numbers, $\left\{w_{i}\right\},\left\{z_{i}\right\}$ being orthonormal systems in $H_{\pi}$.

Then we have

$$
T^{*}=\sum_{i=1}^{k} \bar{\lambda}_{i}\left(\cdot, w_{i}\right) z_{i}
$$

hence

$$
|T|=\left(T^{*} T\right)^{1 / 2}=\sum_{i=1}^{k}\left|\lambda_{i}\right|\left(\cdot, z_{i}\right) z_{i}
$$

and $T=V|T|$, where $V$ is the partial isometry defined by:

$$
z_{i} \rightarrow\left(\lambda_{i} /\left|\lambda_{i}\right|\right) w_{i}, \quad i=1, \ldots, k,
$$

which can be extended to a unitary operator $V^{\prime \prime}$ on $H_{\pi}$. Then $T=V|T|=$ $V^{\prime \prime}|T|$, since

$$
\text { range }(|T|) \subset \operatorname{span}\left(\left\{z_{i}\right\}\right),
$$

so that we have $|T|=U T$, with $U$ unitary, $U=\left(V^{\prime \prime}\right)^{*}$, adjoint of $V^{\prime \prime}$. Now if $\left\{z_{i}\right\}(i=1,2, \ldots, \infty)$ is a completion of the orthonormal system $\left\{z_{i}\right\}$ $(i=1, \ldots, k)$, one has, for every $W \in \mathcal{Q}$ :

$$
\begin{aligned}
\operatorname{tr}(W T) & =\sum_{j=1}^{\infty}\left(W T z_{j}, z_{j}\right) \\
& =\sum_{j=1}^{\infty}\left[W\left[\sum_{i=1}^{k} \lambda_{i}\left(z_{j}, z_{i}\right) w_{i}\right], z_{j}\right] \\
& =\sum_{j=1}^{k}\left(W \lambda_{j} w_{j}, z_{j}\right)=\sum_{j=1}^{k} \lambda_{j}\left(W w_{j}, z_{j}\right) .
\end{aligned}
$$

On the other hand it is easy to see that:

$$
\operatorname{tr}(|T|)=\sum_{i=1}^{k}\left|\lambda_{i}\right|=\|T\|_{1} .
$$

Let $U^{\prime} \in \mathcal{T}$ be such that: $\left|\left(\left[U-U^{\prime}\right] w_{i}, z_{i}\right)\right|<1-\delta, i=1, \ldots, k$. We get 


$$
\begin{aligned}
&\{\sup |\operatorname{tr}(W T)|: W \in \mathscr{V}\}=n(T) \geqslant\left|\operatorname{tr}\left(U^{\prime} T\right)\right| \\
& \quad=\left|\sum_{i=1}^{k} \lambda_{i}\left(\left[U^{\prime}-U\right] w_{i}, z_{i}\right)+\sum_{i=1}^{k} \lambda_{i}\left(U w_{i}, z_{i}\right)\right| \\
& \geqslant\left|\|T\|_{1}-\sum_{i=1}^{k}\right| \lambda_{i}||\left(\left[U^{\prime}-U\right] w_{i}, z_{i}\right)|| \\
& \geqslant\left|\|T\|_{1}-(1-\delta) \sum_{i=1}^{k}\right| \lambda_{i}||=\delta\|T\|_{1} .
\end{aligned}
$$

EXAMPLE 1. Let $\mathcal{Q}$ be the discrete unitary group on the (separable) Hilbert space $H$, and let $\pi$ be the standard representation of $\mathscr{U}$ onto itself; $\pi$ is clearly irreducible, continuous, infinite dimensional if $H$ is so, and $\pi(थ)=$ $\mathcal{Q}$ satisfies trivally the hypothesis of Theorem 1 for every $\delta>0$, so that the two norms are equal on $A_{\pi}$.

Remark 1. In [8, Theorem 4] the closed normal subgroups of the unitary group of a Hilbert space $H$ are characterized. Except for the finite subgroups, they have to contain all those unitary operators which act as the identity on the complement of a finite dimensional subspace, so that they have to be weakly operator dense in the unitary group. Consequently they satisfy the hypothesis of Theorem 1 as well, and the same kind of argument applies to their standard representation in the unitary group as in Example 1.

3. Proof of Theorem 2. In this section we prove Theorem 2, i.e. the inequivalence of the two norms on $A_{\pi}$, for a representation $\pi$ whose coefficients vanish at infinity.

This class includes, for instance, the square integrable representations, since their coefficients may be written as convolutions of two functions in $L^{2}(G)[6$, (14.1)].

LEMMA 1. Let $K$ be a compact subset in a Hilbert space $H,\left\{w_{n}\right\}$ an infinite orthonormal system; then

Proof. By standard arguments.

$$
\lim _{n \rightarrow+\infty}\left\{\sup \left|\left(z, w_{n}\right)\right|: z \in K\right\}=0 .
$$

LEMMA 2. Let $\left\{u_{n}(x)\right\}$ be a sequence of complex continuous functions on a locally compact space $X$ such that:

(i) $\left|u_{n}(x)\right| \leqslant 1$ for every $x \in X, n=0,1,2, \ldots$,

(ii) $u_{n}$ vanishes at infinity for every $n$,

(iii) $\left\{u_{n}\right\}$ tends to zero uniformly on compact sets as $n \rightarrow+\infty$.

Then, given $\varepsilon>0$ and a sequence $\left\{c_{i}\right\} \in l^{2}$ such that

$$
\left\|c_{i}\right\|_{\infty}<\varepsilon / 2\left\|c_{i}\right\|_{2}
$$

there exists a subsequence $\left\{u_{n_{i}}\right\}$ such that

$$
\left\|\sum_{i=0}^{\infty} c_{i} u_{n_{i}}(x)\right\|_{\infty} \leqslant \varepsilon\left\|c_{i}\right\|_{2} .
$$

Here, as usual, $\left\|c_{i}\right\|_{\infty}=\sup \left|c_{i}\right| ;\left\|c_{i}\right\|_{2}=\left\{\sum_{i=0}^{\infty}\left|c_{i}\right|^{2}\right\}^{1 / 2}$. 
Proof. Choose a monotone sequence of positive numbers $\left\{\varepsilon_{i}\right\}$ such that $\left\|\varepsilon_{i}\right\|_{2}<\varepsilon / 2$. Define $n_{0}=0$, and

$$
K_{0}=\left\{x \in X:\left|u_{n_{0}}(x)\right| \geqslant \varepsilon_{0}\right\}
$$

let $n_{1}$ be such that, if $n \geqslant n_{1}$, then $\left|u_{n}(x)\right|<\varepsilon_{1}$ for $x \in K_{n_{0}}$. In general let $n_{i}$ be such that, for $n \geqslant n_{i}$,

$$
\left|u_{n}(x)\right|<\varepsilon_{i} \text { for every } x \in\left(K_{0} \cup K_{1} \cup \cdots \cup K_{i-1}\right)
$$

and

$$
K_{i}=\left\{x \in X:\left|u_{n_{i}}(x)\right| \geqslant \varepsilon_{i}\right\}
$$

Notice that the $K_{i}$ 's are compact by (ii) and disjoint; for, if $x \in K_{j}$, then $\left|u_{n_{j}}(x)\right| \geqslant \varepsilon_{j}$, hence $x$ cannot belong to $K_{i}$ for $i<j$. Now, if $x \notin \cup_{i=0}^{\infty} K_{i}$, then $\left|u_{n_{i}}(x)\right|<\varepsilon_{i}$ for every $i$, and

$$
\left|\sum_{i=0}^{\infty} c_{i} u_{n_{i}}(x)\right| \leqslant \sum_{i=0}^{\infty}\left|c_{i}\right|\left|u_{n_{i}}(x)\right| \leqslant \sum_{i=0}^{\infty} \varepsilon_{i} c_{i} \leqslant\left\|\varepsilon_{i}\right\|_{2}\left\|c_{i}\right\|_{2}<\varepsilon / 2\left\|c_{i}\right\|_{2} .
$$

If $x \in \cup_{\mathrm{i}=0}^{\infty} K_{i}$, then there exists exactly one $j$ such that $x \in K_{j}$; then, by (i):

$$
\begin{aligned}
\left|\sum_{i=0}^{\infty} c_{i} u_{n_{i}}(x)\right| & \leqslant\left\{\sum_{i \neq j}\left|c_{i}\right|\left|u_{n_{i}}(x)\right|+\left|c_{j}\right|\left|u_{n_{j}}(x)\right|\right\} \\
& \leqslant \varepsilon / 2\left\|c_{i}\right\|_{2}+\varepsilon / 2\left\|c_{i}\right\|_{2} .
\end{aligned}
$$

Proof of Theorem 2. Given $\varepsilon>0$, choose an infinite orthonormal system $\left\{w_{n}\right\}$ and a sequence of numbers $\left\{c_{i}\right\}$ as in Lemma 2. Define

$$
u_{n}(x)=\left(\pi(x) w_{n}, w_{0}\right) \text {. }
$$

It is straightforward to see that the sequence $\left\{u_{n}\right\}$ satisfies (i) and (ii) of Lemma 2, while (iii) follows from the identity

$$
\left(\pi(x) w_{n}, w_{0}\right)=\left(w_{n}, \pi^{*}(x) w_{0}\right)
$$

Lemma 1 and the continuity of $\pi$.Therefore we may find a subsequence $\left\{u_{n_{i}}\right\}$ satisfying the conclusion of Lemma 2 . Define the operator $T$ by:

$$
T=\sum_{i=0}^{\infty} c_{i}\left(\cdot, w_{0}\right) w_{n_{i}} .
$$

An easy computation shows that $\operatorname{tr}(|T|)=\left\|c_{i}\right\|_{2}$. Moreover:

$$
u(x)=t(x)=\operatorname{tr}(\pi(x) T)=\sum_{i=0}^{\infty} c_{i}\left(\pi(x) w_{n_{i}}, w_{0}\right) .
$$

Then by Lemma 2

$$
\|u\|_{\infty} \leqslant \varepsilon\left\|c_{i}\right\|_{2}=\varepsilon \operatorname{tr}(|T|)=\varepsilon\|u\|_{B} .
$$

Since $\varepsilon$ is arbitrary the two norms are inequivalent.

REMARK 2 . This argument can actually be carried out whenever we have a family $\mathcal{G}$ of subsets of $G$ such that:

(i) $\mathcal{G}$ is stable under finite unions,

(ii) there exists an orthonormal system $\left\{w_{n}\right\}$ in the Hilbert space $H_{\pi}$ such 
that the sequence of coefficients

$$
u_{n}(x)=\left(\pi(x) w_{n}, w_{0}\right)
$$

goes to zero uniformly on the sets of $\mathcal{G}$,

(iii) the coefficients $u_{n}(x)$ "vanish at infinity" with respect to $\mathcal{G}$, i.e. for every $\varepsilon>0$ the set $\left\{x \in G:\left|u_{n}(x)\right| \geqslant \varepsilon\right\}$ belongs to $\mathcal{G}$.

REMARK 3. If we replace the topology of $G$ with a finer one (e.g. the discrete topology) we may get a representation whose coefficients do not vanish at infinity, while the two norms are still inequivalent, for $A_{\pi}$ does not change [2, (2.11)], [1].

REMARK 4. By. the functorial properties of the space $A_{\pi},[2,(2.10)]$ (see also $[1,(2.20)])$, Theorem 2 holds for representations whose coefficients vanish at infinity modulo the kernel.

4. Proof of Theorem 3. (We assume, for simplicity, the separability of the Hilbert space $H_{\rho}$; the group $G$ is not necessarily separable.)

Let us recall that the space $H_{\pi}$ of the induced representation $\pi$ is the space of (the equivalence classes of) the functions $\phi: G \rightarrow H_{\rho}$ such that:

(i) $\phi(x n)=\rho^{*}(n) \phi(x)$ for every $x \in G, n \in N$,

(ii) $(\phi(x), z)$ are measurable functions of $x$ for every $z \in H_{\rho}$,

(iii)

$$
\|\phi\|_{H_{\pi}}=\left\{\int_{G / N}\left\|\phi\left(x^{\prime}\right)\right\|_{H_{\rho}}^{2} d \mu\left(x^{\prime}\right)\right\}^{1 / 2}<+\infty,
$$

$x^{\prime}$ being the equivalence class of $x$ in $G / N$ and $\mu$ the relatively invariant measure on $G / N$ [5, Chapters V, VI].

The inner product in the space $H_{\pi}$ is

$$
(\phi, \psi)=\int_{G / N}(\phi(x), \psi(x))_{H_{\rho}} d \mu\left(x^{\prime}\right) .
$$

If $\lambda(g), g \in G$, is the modular function of the relatively invariant measure $\mu$ on $G / N$, we have:

$$
[\pi(g) \phi](x)=\phi\left(g^{-1} x\right)\left\{\lambda\left(g^{-1}\right)\right\}^{1 / 2} .
$$

Let us consider a continuous, nonnegative real valued function $f$ on $G$, whose support $S$ is such that $S \cap C N$ is compact for every compact $C$ in $G$, and which satisfies the relation

$$
\int_{N} f(x n) d n=1 \text { for every } x \in G,
$$

$d n$ being the left Haar measure on $N$ [5, p. 258].

Let the function $f^{\#}: G \rightarrow H_{\rho}$ be defined by:

$$
f^{\sharp}(x)=\int_{N} \rho(n) w f(x n) d n,
$$

$w$ being a fixed unitary vector in $H_{\rho}$; then $f^{\sharp}$ is continuous, satisfies condition (i), and $\left\|f^{\sharp}(x)\right\|_{H_{\rho}} \leqslant 1$ for every $x \in G$. This follows from [5, Lemma 3, p. 260] and [5, Lemma 2, p. 372] combined. Moreover $f^{\sharp}(e)=\hat{\rho}\left(\left.f\right|_{N}\right) w$, where $e$ 
is the unit of $G$ and $\rho^{\wedge}$ is the representation of $L^{1}(N)$ canonically associated to the representation $\rho$ of $N$; therefore the three above properties of $f^{\sharp}$ ensure that, if $S \cap N$ is chosen small enough, then $f^{\#}$ is different from zero in an open saturated neighborhood of $e$. Let $\left\{E_{j}\right\}, j=0,1, \ldots$, be a sequence of compact sets in $G / N$ with the following properties:

(a) $\mu\left(E_{j}\right) \rightarrow 0$ as $j \rightarrow+\infty$,

(b) $E_{0} \cap E_{j}=\varnothing$ and $E_{j+1} \subset E_{j}$ for $j=1,2, \ldots$,

(c) the saturated subsets of $G: F_{j}=p^{-1}\left(E_{j}\right)$ are contained in the open saturated set in $G$ where the function $f^{\#}$ is not zero. ( $p$ denotes the projection $G \rightarrow G / N$.)

If we define

$$
\phi_{j}(x)=\left\{\int_{E_{j}}\left\|f^{\sharp}\left(x^{\prime}\right)\right\|^{2} d \mu\left(x^{\prime}\right)\right\}^{-1 / 2} \chi_{j}(x) f^{\sharp}(x)
$$

where $\chi_{j}$ is the characteristic function of the set $F_{j}$, it is easy to see that the functions $\phi_{j}$ belong to the space $H_{\pi}$ and that $\left\|\phi_{j}\right\|=1$. If $T_{j}$ is the rank one operator on $H_{\pi}$ defined by:

$$
T_{j}(\psi)=\left(\psi, \phi_{0}\right) \phi_{j}
$$

then $\left\|t_{j}\right\|_{B}=\left\|T_{j}\right\|=1$ for every $j$. (See Proposition 1.) It only remains to show that $\left\|t_{j}\right\|_{\infty} \rightarrow 0$ as $j \rightarrow+\infty$.

Routine calculations and the properties of $f^{\sharp}$ show that

$$
\left|t_{j}(g)\right| \leqslant\left\{\lambda(g) \mu\left(E_{j} \cap\left(g^{-1} E_{0}\right)\right)\right\}^{1 / 2} \text {. }
$$

If $\mu$ is $G$-invariant, $\lambda(g)=1^{\prime}$ for every $g \in G$ and $\mu\left(E_{j} \cap\left(g^{-1} E_{0}\right)\right)$ is dominated by $\mu\left(E_{j}\right)$ uniformly with respect to $g \in G$, hence $\left\|t_{j}\right\|_{\infty} \rightarrow 0$. Otherwise it is easy to see that

$$
Z=\left\{g: E_{j} \cap\left(g^{-1} E_{0}\right) \neq \varnothing\right\}=C_{j} N C_{0}^{-1},
$$

where $C_{j}$ and $C_{0}$ are compact sets in $G$ such that $p\left(C_{j}\right)=E_{j}$. We have $\lambda(g) \leqslant$ constant for $g \in C_{j} N C_{0}^{-1} \subset C_{1} N C_{0}^{-1}$, since $N$ is compact and $\lambda$ continuous, being the modular function of a relatively invariant measure, and this completes the proof.

\section{REFERENCES}

1. P. Eymard, L'algèbre de Fourier d'une groupe localement compact, Bull. Soc. Math. France 92 (1964), 181-236.

2. G. Arsac, Sur l'espace de Banach engendré par les coefficients d'une représentation unitaire (Doctoral thèse, Université Claude-Bernard), Publ. Dép. Math. (Lyon) 13 (1976), 1-101.

3. , Sur un espace fonctionnel associé à une représentation unitaire d'un groupe localement compact, C. R. Acad. Sci. Paris 273 (1971), 298-300.

4. W. Rudin, Fourier analysis on groups, Interscience Tracts in Math., no. 7, Interscience, New York, 1962.

5. S. A. Gaal, Linear analysis and representation theory, Springer-Verlag, Berlin, 1973.

6. J. Dixmier, Les $C^{*}$-algèbres et leurs représentations, Gauthier-Villars, Paris, 1964.

7. M. E. Walter, On the structure of the Fourier-Stieltjes algebra, Pacific J. Math. 58 (1975), 267-281.

8. R. V. Kadison, Infinite unitary groups, Trans. Amer. Math. Soc. 72 (1952), 386-399.

Istituto Matematico Universitá di Milano, vi Saldini 50, 20133 Milano, Italy 